\title{
Modified Euler integration based control of a five-phase induction motor drive
}

\author{
M. Rizwan Khan ${ }^{1 *}$, Atif Iqbal ${ }^{1,2}$, SK. Moin Ahmed ${ }^{1}$, Saifullah Payami \\ ${ }^{1^{*}}$ Department of Electrical Engineering, Aligarh Muslim University, Aligarh, INDIA \\ ${ }^{2}$ Department of Electrical Engineering, College of Engineering, Qatar University, Doha, QATAR \\ ${ }^{*}$ Corresponding Author: e-mail: rizwan.eed@gmail.com, Tel +91-9412545798, Fax.+91-571-2721178
}

\begin{abstract}
In principle, control methods for multi-phase machines are the same as for three-phase machines. Variable speed induction motor drives without mechanical speed sensors at the motor shaft have the attractions of low cost and high reliability. To replace the speed sensor, information of the rotor speed is extracted from measured stator currents and voltages at motor terminals. Vector-controlled drives require estimating the magnitude and spatial orientation of the fundamental magnetic flux in the stator or in the rotor. Open-loop estimators or closed-loop observers are used for this purpose. They differ with respect to accuracy, robustness, and sensitivity against model parameter variations. This paper analyses operation of a modified Euler integrationbased sensorless control of vector controlled five-phase induction machine with current control in the stationary reference frame. A linear neural network has been then designed and trained online by means of back propagation network (BPN) algorithm, differently from that in the literature which employs a nonlinear back propagation network (BPN) algorithm. The Artificial Neural Network (ANN)-Model Reference Adaptive System (MRAS) based sensorless operation of a three-phase induction machine is well established and the same principle is extended in this paper for a five-phase induction machine. Performance, obtainable with hysteresis current control, is illustrated for a number of operating conditions on the basis of simulation results. Full decoupling of rotor flux control and torque control is realised. Dynamics, achievable with a five-phase vector controlled induction machine, are shown to be essentially identical to those obtainable with a three-phase induction machine.
\end{abstract}

Keywords: Multi-phase machine, MRAS, ANN, BPN, Euler Integration, Sensorless control.

\section{Introduction}

Variable speed electric drives predominately utilise the three-phase machines. However, since the variable speed ac drives require a power electronic converter for their supply (in vast majority of cases an inverter with a dc link), the number of machine phases is essentially unlimited. This has led to an increase in the interest in multiphase ac drive applications, since multiphase machines offer some inherent advantages over their three-phase counterparts. a number of interesting research results has been published over the years and detailed reviews are available in (Singh, 2002, Jones et al., 2002, Toliyat et al., 2000, Xu et al., 2002, Parsa, 2005).

Major advantages of using a multi-phase machine instead of a three-phase machine are detailed in (Singh, 2002, Jones et al., 2002, Toliyat et al., 2000, Xu et al., 2002, Parsa, 2005) and are higher torque density, greater efficiency, reduced torque pulsations, greater fault tolerance, and reduction in the required rating per inverter leg (and therefore simpler and more reliable power conditioning equipment). Additionally, noise characteristics of the drive improve as well.

Sensorless vector control of three-phase induction machine has attracted wide attention in resent years (Parsa et al, 2004, Terrien et al., 2004, Holtz, 2006, 2002). Several attempts have been made in the past to extract the speed signal of the induction machine from measured stator currents and voltages. Initially, the sensorless techniques were restricted to techniques which are only valid in the steady-state and can only be used in low cost drive applications, not requiring high dynamic performance. Different, more sophisticated techniques are required for high performance applications in vector controlled drives (Holtz, 2002). In a sensorless drive, speed information and control should be provided with an accuracy of $0.5 \%$ or better, from zero to the highest speed, for all 
operating conditions and independent of saturation levels and parameter variations. In order to achieve good performance of sensorless vector control, different speed estimation schemes have been proposed and a variety of speed estimators exist nowadays.

Sensorless operation of a vector controlled three-phase induction machine drive is extensively discussed in the literature (Vas, 1998, Rajashekara et al, 1996), but the same is not true for multi-phase induction machine. Only few application specific sensorless operation of multi-phase machine is elaborated in the literature. The problem of using the position sensor in 'moreelectric' aircraft fuel pump fault tolerant drive is highlighted in (Green et al, 2000). The drive utilises a $16 \mathrm{~kW}, 13000 \mathrm{rpm}$ sixphase permanent magnet motor with six independent single-phase inverters supplying each of the six-phases. The authors proposed an alternative sensorless drive scheme. The proposed technique makes use of flux linkage-current-angle model to estimate the rotor position.

Although several schemes are available for sensorless operation of a vector controlled drive, but the most popular is the MRAS because of ease of their implementation (Elbulk et al, 2002, Kim et al, 1995). An attempt is made in this paper to extend the MRAS-based technique of a three-phase machine to an indirect field oriented five-phase induction motor drive.

It has been shown in (White, 1959) that multi-phase machine models can be transformed into a system of decoupled equations in orthogonal reference frames. The $d-q$ axis reference frame currents contribute towards torque and flux production, whereas the remaining $x-y$ components plus the zero sequence components do not. This allows a simple extension of the rotor flux oriented control(RFOC) principle in that the rotor flux linkage is maintained entirely in the $d$-axis, resulting in the $q$-axis component of rotor flux being maintained at zero. This reduces the electromagnetic torque equation to the same form as that of a dc machine or a rotor flux oriented three-phase machine. Thus the electromagnetic torque and the rotor flux can be controlled independently, by controlling the $d$ and $q$ components of stator current independently. The decoupled control of torque and flux using rotor flux oriented control for a five-phase induction machine is illustrated in (Xu et al, 2002).

Ben et al. (1993) and Elloumi et al. (1998) present an MRAS speed observer which is an evolution of Shauder (1992) and minimizes the error between rotor fluxes estimated respectively with a reference and an adaptive flux model, and then they apply it to an field oriented control(FOC). Like in Shauder (1992), it employs, as a reference model, the voltage model of the induction machine and the open-loop integration is performed by an LP filter. However, it uses the adaptive model, by rearranging the rotor equations of the machine so that a multilayer perceptron can be employed. On this basis, these articles exploit the classical back propagation network (BPN) algorithm for the online training of the neural network to estimate the rotor speed. In Ben et al. (1999) the observer is verified also experimentally, even if neither the lowest speed limit of the observer nor the zero-speed operations, at no load and at load, are presented. This paper proposes an improvement of the MRAS artificial-neural-network (ANN)-based speed observer presented in (Ben et al, 1999), for basically two reasons. First it does not use the BPN neural network but an Adaptive Linear neural Network (ADALINE), since the problem to be solved is linear: it is in fact questionable to use a nonlinear method like the BPN algorithm which causes local minima, paralysis of the neural network, need of two heuristically chosen parameters, initialization problems, and convergence problems. In (Ben et al, 1999) this linearity problem has been recognized, but the minimization has been performed with a gradient-descent dependent also from the momentum, which is not necessary. Second, the adaptive model in (Ben et al, 1999) is used in simulation mode, which means that its outputs are feed back recursively. In this paper, in contrast to this, a modified adaptive model is used as a predictor, without feedbacks, no need of filtering the estimated signal, and resulting in higher accuracy both in transient and steady-state operation. Moreover, differently from (Ben et al, 1999), a stable behavior in field weakening is achieved. In this paper the improvements achieved with the MRAS-OLS observer are emphasized.

The analysis is here restricted to ANN-MRAS-based sensorless control of a five-phase induction machine, with current control in the stationary reference frame. The ordinary least square (OLS) algorithm for the online training of the neural network is used to estimate the rotor speed. Phase currents are controlled using hysteresis current control method. A simulation study is performed for speed mode of operation, for a number of transients, and the results are reported in the paper.

A detailed modelling of a five-phase induction machine and it vector control principle is reported in (Jones et al, 2002, Xu et al, 2002).

The developed model of a five-phase induction motor indicates that an ANN-MRAS technique used for three-phase machines can be easily extended to multi-phase machines. For multi-phase machines ANN-MRAS-based speed estimator requires only $d$ and $q$ components of stator voltages and currents. The model of a five-phase induction machine (Jones et al, 2002, Xu et al, 2002), it has been shown that the stator and rotor $d$ and $q$ axis flux linkages are function of magnetising inductance $L m$ and stator and rotor $d$ and $q$ axis currents, where as the $x$ and $y$ axis flux linkages are function of only their respective currents. Therefore in speed estimation for multi-phase machine the $x$ and $y$ components of voltages and currents are not required. The speed can be estimated using only $d$ and $q$ components of stator voltages and currents.

The proposed ANN-MRAS-based five-phase vector controlled induction motor drive structure with current control in the stationary reference frame is shown in Figure 1. 


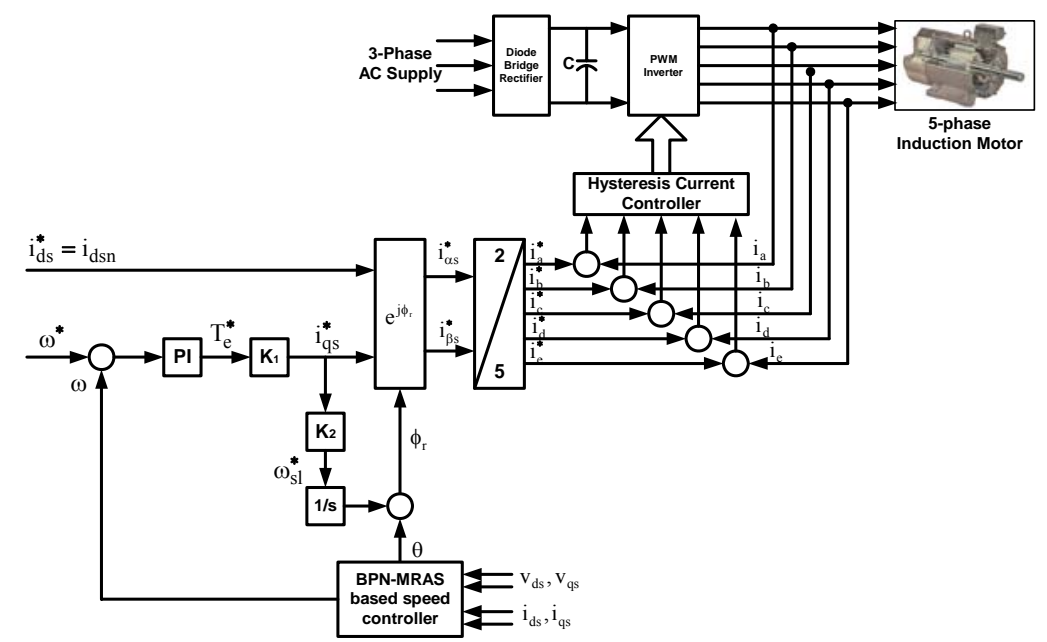

Figure 1. ANN MRAS BPN based five-phase induction motor drive structure.

\section{BPN Algorithm based Speed Estimator}

\section{(A) Using Simple Euler Integration Method}

A linear neural network has been designed and trained online by means of BPN algorithm. It is clear from "Figure 2(a)," that the adaptive model is characterized by the feedback of delayed estimated rotor flux components to the input of the neural network, which means that the adaptive model employed is in simulation mode. Moreover, the adaptive model is tuned online (training) by means of a BPN algorithm, which is, however, nonlinear in its nature with the consequent drawbacks (local minima, heuristics in the choice of the network parameters, paralysis, convergence problems, and so on).

In Figure 2(b), the adaptive model employs an ADLINE and the values of the rotor flux-linkage components at the input of the ANN come from the reference model, and not from the adaptive one; this means that the ANN is employed in prediction mode.

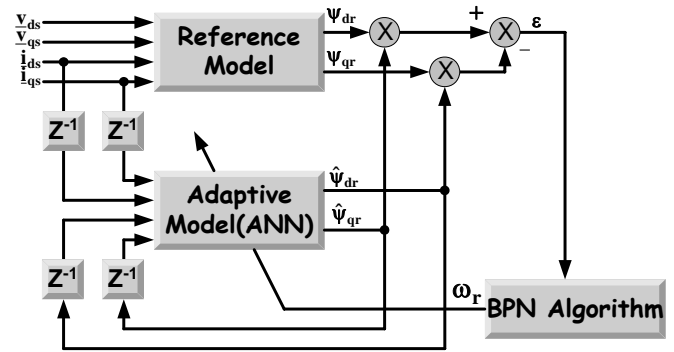

(a)

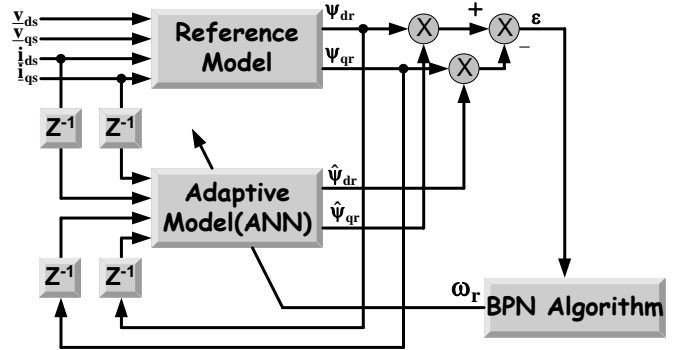

(b)

Figure 2. Schematic block diagram of Recurrent Neural Network speed estimator using BPN algorithm in

(a) Simulation mode and (b) Prediction mode.

Figure 2(a) and Figure 2(b) show the block diagrams of MRAS-based speed estimation schemes, which contain an ANN. This network has four inputs and two outputs. It follows from these figures that the inputs to the reference model are the monitored stator voltages and currents of the induction machine. The outputs of the reference model are the rotor flux-linkage components in the stationary reference frame $\left(\psi_{r d}, \psi_{r q}\right)$. These are obtained by considering the following equations (Vas, 1998):

$$
\begin{aligned}
& \psi_{d r}=\frac{L_{r}}{L_{m}}\left[\int\left(V_{d s}-R_{s} i_{d s}\right) d t-L_{s}{ }^{\prime} i_{d s}\right] \\
& \psi_{q r}=\frac{L_{r}}{L_{m}}\left[\int\left(V_{q s}-R_{s} i_{q s}\right) d t-L_{s}{ }^{\prime} i_{q s}\right]
\end{aligned}
$$

These two equations do not contain the rotor speed and describe the reference model. However, when the rotor voltage equations of the induction machine are expressed in the stationary reference frame, they contain the rotor flux linkages and the rotor speed as well. These are the equations of the adaptive model and are given as:

$$
\hat{\psi}_{d r}=\frac{1}{T_{r}}\left[\int\left(L_{m} i_{d s}-\hat{\psi}_{d r}-\omega_{r} T_{r} \hat{\psi}_{q r}\right) d t\right]
$$




$$
\hat{\psi}_{q r}=\frac{1}{T_{r}}\left[\int\left(L_{m} i_{q s}-\hat{\psi}_{q r}+\omega_{r} T_{r} \hat{\psi}_{d r}\right) d t\right]
$$

In these equations $\hat{\psi}_{d r}$ and $\hat{\psi}_{q r}$ are the rotor flux linkages estimated by the adaptive model. Equations (3) and (4) contain the rotor speed, which in general is changing, and it is our purpose to estimate this speed by using an ANN. For this purpose, Equations (3) and (4) can be implemented by a two-layer ANN which contains variable weights, and the variable weights are proportional to the rotor speed

For given stator voltages and currents and induction machine parameters, the actual rotor speed $\omega_{r}$ must be the same as the speed estimated by the ANN $\left(\hat{\omega}_{r}\right)$, when the outputs of the reference model and the adaptive model are equal. In this case the errors $\varepsilon_{d}=\psi_{d r}-\hat{\psi}_{d r}$ and $\varepsilon_{q}=\psi_{q r}-\hat{\psi}_{q r}$ are zero.

When there is any mismatch between the rotor speed estimated by the ANN and the actual rotor speed, then these errors are not zero, and they are used to adjust the weights of the ANN (or in other words the estimated speed). The weight adjustment is performed in such a way that the error should converge fast to zero.

To obtain the required weight adjustments in the ANN, the sampled data forms of equations (3) and (4) are considered. Using the backward difference method, e.g. considering the rate of change of an estimated rotor flux linkage

$$
\frac{d \hat{\psi}_{d r}}{d t}=\frac{\hat{\psi}_{d r}(k)-\hat{\psi}_{q r}(k-1)}{T_{s}}
$$

Where $T_{s}$ is the sampling time, the sampled data forms of the equations for the rotor flux linkages can be written as:

$$
\begin{aligned}
& \frac{\hat{\psi}_{d r}(k)-\hat{\psi}_{d r}(k-1)}{T_{s}}=-\frac{\hat{\psi}_{d r}(k-1)}{T_{r}}-\frac{\omega_{r} \hat{\psi}_{q r}(k-1)}{T_{s}}+\frac{L_{m}}{T_{r}} i_{d s}(k-1) \\
& \frac{\hat{\psi}_{q r}(k)-\hat{\psi}_{q r}(k-1)}{T_{s}}=-\frac{\hat{\psi}_{q r}(k-1)}{T_{r}}+\frac{\omega_{r} \hat{\psi}_{d r}(k-1)}{T_{s}}+\frac{L_{m}}{T_{r}} i_{q s}(k-1)
\end{aligned}
$$

Thus the rotor flux linkages at the $\mathrm{k}^{\text {th }}$ sampling instant can be obtained from the previous $(\mathrm{k}-1)^{\text {th }}$ values as

$$
\begin{aligned}
& \hat{\psi}_{d r}(k)=\left(1-\frac{T}{T_{r}}\right) \hat{\psi}_{d r}(k-1)-w_{r} T_{s} \hat{\psi}_{q r}(k-1)+\frac{L_{m} T_{s}}{T_{r}} i_{d s}(k-1) \\
& \hat{\psi}_{q r}(k)=\left(1-\frac{T}{T_{r}}\right) \hat{\psi}_{q r}(k-1)+w_{r} T_{s} \hat{\psi}_{d r}(k-1)+\frac{L_{m} T_{s}}{T_{r}} i_{q s}(k-1)
\end{aligned}
$$

By introducing $\mathrm{c}=T_{s} / T_{r}$ and assuming that the rotor time constant $\left(T_{r}\right)$ is constant, the following weights are introduced:

$$
w_{1}=1-c, w_{2}=\omega_{r} T_{s}, w_{3}=c L_{m} \text { and } c=\frac{T_{s}}{T_{r}}
$$

Where $T_{r}=\frac{L_{r}}{R_{r}}, \varepsilon_{d}=\psi_{d r}-\hat{\psi}_{d r}$ and $\varepsilon_{q}=\psi_{q r}-\hat{\psi}_{q r}$

It can be seen that $\omega_{1}$, and $\omega_{3}$ are constant weights, but $\omega_{2}$ is a variable weight, and is proportional to the speed. Thus equations (8) and (9) take the following forms:

$$
\begin{aligned}
& \hat{\psi}_{d r}(k)=w_{1} \hat{\psi}_{d r}(k-1)-w_{2} \hat{\psi}_{q r}(k-1)+w_{3} i_{d s}(k-1) \\
& \hat{\psi}_{q r}(k)=w_{1} \hat{\psi}_{q r}(k-1)-w_{2} \hat{\psi}_{d r}(k-1)+w_{3} i_{q s}(k-1)
\end{aligned}
$$

These equations can be visualized by the very simple two-layer ANN shown in "Figure 1,". This contains four input nodes. The input signals to these input nodes are the past values of the estimated rotor flux-linkage components expressed in the stationary reference frame $\left[\hat{\psi}_{d r}(k-1), \hat{\psi}_{q r}(k-1)\right]$, and also the past values of the stator current components expressed in the stationary reference frame $\left[i_{d s}(k-1), i_{q s}(k-1)\right]$. There are two output nodes which output the present values of the estimated rotor flux- 
linkage components $\left[\hat{\psi}_{d r}(k), \hat{\psi}_{q r}(k)\right]$. Thus all the nodes are well defined. The connections between the nodes are represented by weights (synapses), and a weight shows the strength of the connection considered. In general a weight can be positive or negative, corresponding to excitatory and inhibitory weights.

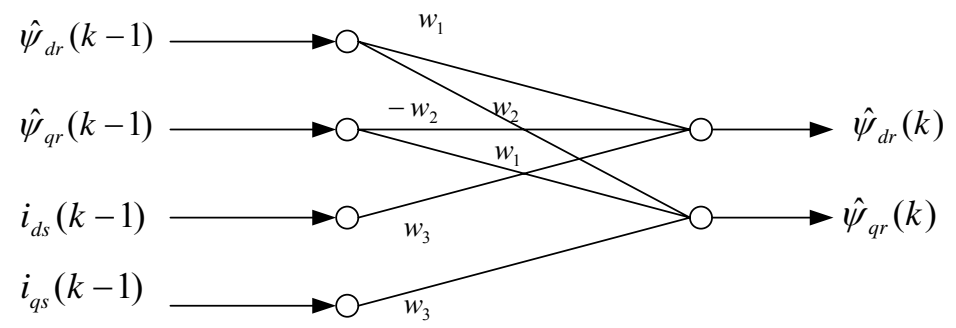

Figure 3. ANN representation of estimated rotor flux linkages.

In the ANN shown in Figure 3, the adaptive $w_{2}$ weights are shown with thick solid lines and, as noted above, these are proportional to the speed $\left(w_{2}=\omega_{r} T_{s}\right)$ where the proportionality factor is the sampling time. The adaptive weights are adjusted so that $E=(1 / 2) \varepsilon^{2}(k)$ should be a minimum, where,

$\varepsilon(k)=\psi_{r}(k)-\hat{\psi}_{r}, \psi_{r}(k)=\left[\psi_{d r}(k), \psi_{q r}(k)\right]^{T}$ and $\hat{\psi}_{r}(k)=\left[\hat{\psi}_{d r}(k), \hat{\psi}_{q r}(k)\right]^{T}$

Thus the weight adjustments to give minimum squared error have to be proportional to the negative of the gradient of the error with respect to the weight, $-\partial E / \partial w_{2}$ since in this way it is possible to move progressively towards the optimum solution, where the squared error is minimal. The proportionality factor is the so-called learning rate, $\eta$. which is a positive constant and larger learning rates yield larger changes in the weights. In practice as large a value is chosen for the learning rate as possible, since this gives the fastest learning, but a large learning rate can yield oscillations in the output of the ANN.

It follows from the above that the mathematical expression for the weight adjustment has to be

$$
\Delta w_{2}(k)=-\eta \partial E / \partial w_{2}
$$

This after simplification gives the following expression:

$$
\Delta w_{2}(k)=\eta\left[-\varepsilon_{d}(k) \hat{\psi}_{q r}(k-1)+\varepsilon_{q}(k) \hat{\psi}_{d r}(k-1)\right]
$$

Where $\varepsilon_{d}(k)=\psi_{d r}(k)-\hat{\psi}_{d r}(k)$, and $\varepsilon_{q}(k)=\psi_{q r}(k)-\hat{\psi}_{q r}(k)$. Equation (15) is a well known type of expression in neural networks using the method of steepest $\&$ gradient for weight adjustment and it can be seen that the appropriate errors are multiplied by the appropriate inputs of the neural network shown in Figure 3.

Thus in Figure 3, the weight adjustments can be obtained from

$$
w_{2}(k)=w_{2}(k-1)+\Delta w_{2}(k)
$$

It has been discussed above that for rapid learning, the learning rate $(\eta)$ has to be selected to be large, but this can lead to oscillations in the outputs of the ANN. However, to overcome this difficulty, a so-called momentum term is added to Equation (16). Which takes into account the past $\left[(\mathrm{k}-1)^{\text {th }}\right]$ weight changes on the present $\left[\mathrm{k}^{\text {th }}\right]$ weight This ensures accelerated convergence of the algorithm. Thus the current weight adjustment $\Delta w_{2}(k)$ described by Equation (16) is supplemented by a fraction of the most recent weight adjustment, $\Delta w_{2}(k-1)$ :

$$
w_{2}(k)=w_{2}(k-1)+\Delta w_{2}(k)+\alpha \Delta w_{2}(k-1)
$$

Where $\alpha$ is a positive constant called the momentum constant. The term $\alpha . \Delta w_{2}(k-1)$ is called the momentum term, and is a scaled value of the most recent weight adjustment. Usually, $\alpha$ is in the range between 0.1 and 0.8 . The inclusion of the momentum term into the weight adjustment mechanism can significantly increase the convergence, which is extremely useful when the ANN shown in Figure 3, is used to estimate in real-time the speed of the induction machine. Since it follows from equation $w_{2}=\omega_{r} T_{s}$, that the weight $\omega_{2}$ is proportional to the speed, finally the estimated rotor speed can be obtained from

$$
\omega_{r}=\frac{W_{2}}{T_{s}}
$$

\section{Simulation Results and Discussion}

The adaptation algorithm described above can be implemented in two ways (i) ANN MRAS BPN Model (adaptive model in Simulation mode), and (ii) ANN MRAS BPN Model (adaptive model in Prediction mode). It is clear from the Figure 1, 
that the adaptive model is characterized by the feedback of delayed estimated rotor flux components to the input of the neural network, which means that the adaptive model employed is in simulation mode. Moreover, the adaptive model is tuned online (training) by means of a BPN algorithm, which is nonlinear in nature. In the second mode Figure 2, the values of the rotor flux-linkage components at the input of the ANN come from the reference model, and not from the adaptive one; this means that the ANN is employed in prediction and not in simulation mode.

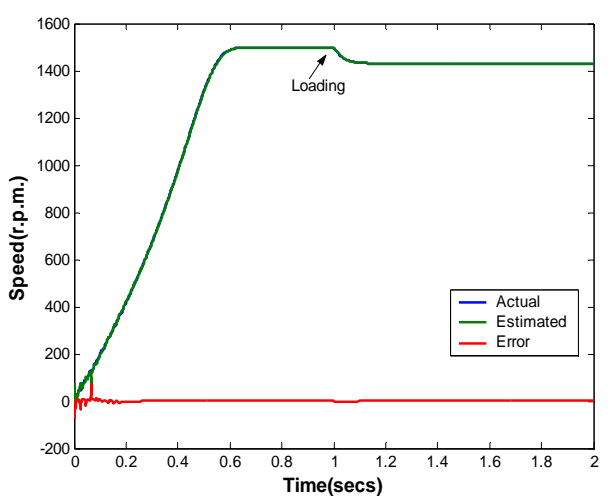

(a)

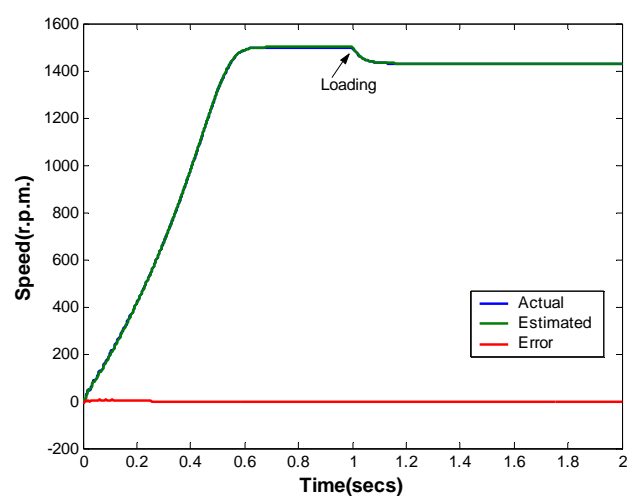

(b)

Figure 4. Estimated Speed of the ANN-MRAS-BPN scheme (a) adaptive model in Simulation mode (b) adaptive model in prediction mode

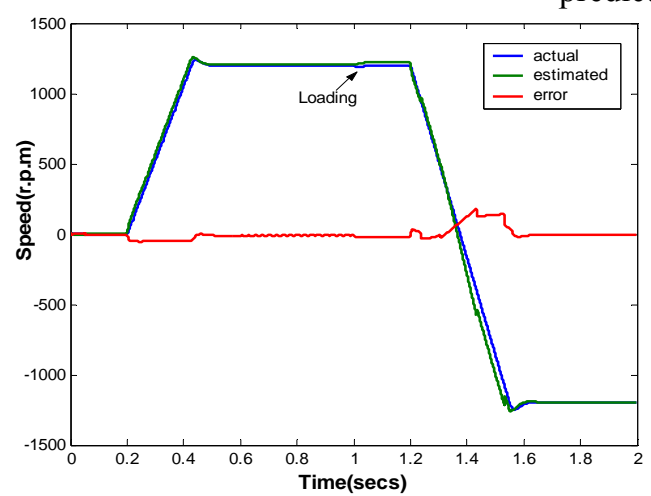

(a)

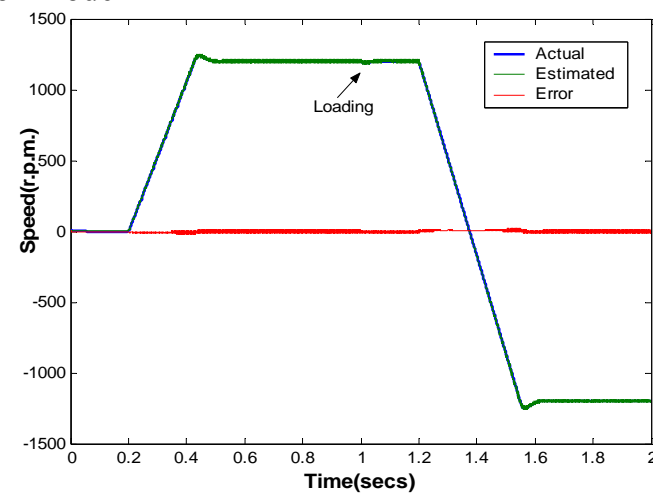

(b)

Figure 5. Speed response with vector controller of the ANN-MRAS-BPN scheme (a) adaptive model in Simulation mode (b) adaptive model in prediction mode.

It is observed from the simulation results (Figures 4 and Figure 5) that much better response is obtained if it is implemented in the prediction mode the starting ripples which are the problem of ANN speed estimator can be eliminated $\&$ the tracking capability further improves in the prediction mode. The neural adaptive model employed in prediction mode, has the advantages that it gives quicker convergence of the speed estimation, higher bandwidth of the speed loop, lower estimation errors both in transient and steady-state operation, better behaviour in zero-speed operation at no load, and stable behaviour in field weakening.

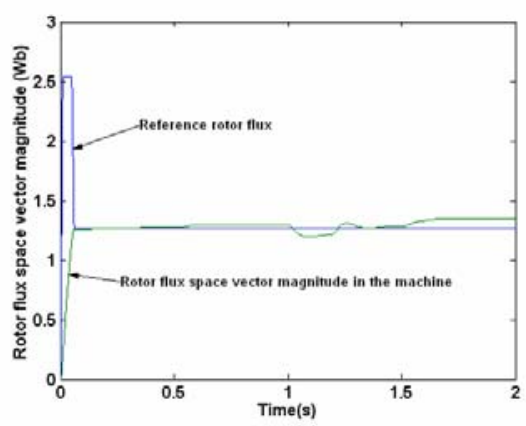

(a)

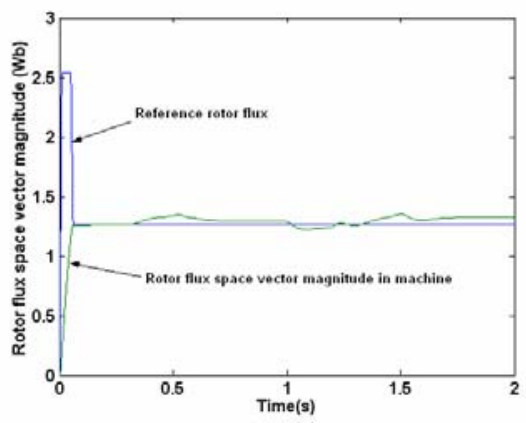

(b)

Figure 6. Actual and reference rotor flux for (a) Simulation mode (b) Prediction mode. 

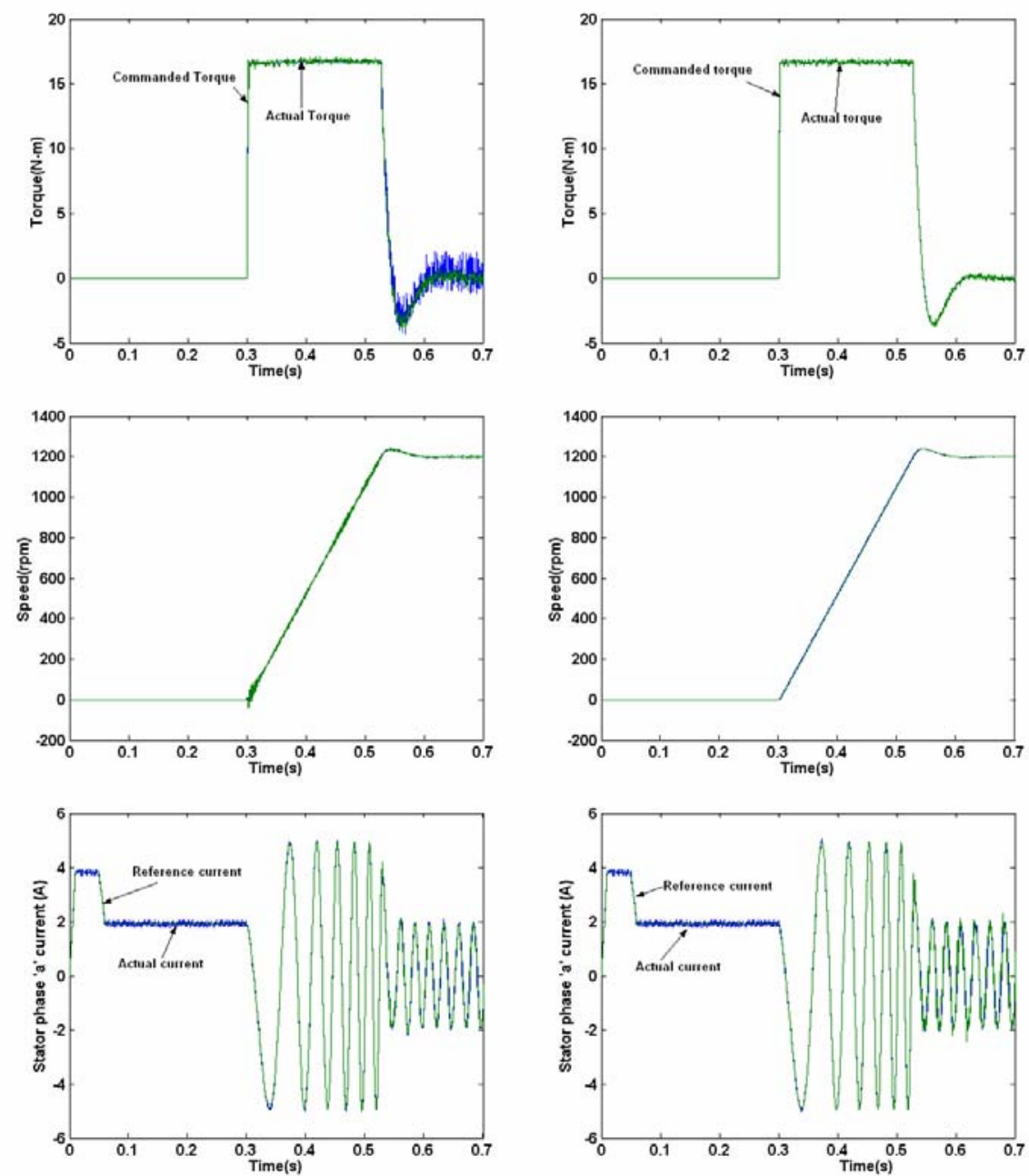

(a)

(b)

Figure 7. The torque, speed and phase ' $a$ ' current of a vector controlled five-phase induction machine: excitation and acceleration transient for (a) simulation mode and (b) prediction mode.

The drive is operated in speed mode with speed feedback is taken from the BPN-MRAS speed estimator. Figure 6 displays results for reference and actual rotor flux in simulation mode and prediction mode. After the initial transient rotor flux settles to the reference value and there is a change of flux of $0.15 \mathrm{~Wb}$ in simulation mode and $0.13 \mathrm{~Wb}$ in prediction mode. It is due to loss of decoupled control or parameter variation effect or lack of proper tuning. Figure 7 displays results for reference and actual torque, speed, stator phase ' $a$ ' reference and actual current and stator phase 'a' phase-to-neutral voltage for both modes. It can be seen from Figure 7 that the flux and torque control are fully decoupled. Acceleration takes place with the maximum allowed value of the motor torque. Actual motor phase current tracks the reference very well. Consequently, torque response closely follows torque reference and a small deviation appears only at the end of the transient. No viable conclusion can be drawn because of PWM nature of voltages. In simulation mode, the ripples in the torque are of $4 \mathrm{~N}-\mathrm{m}$ and spikes in speed are of $80 \mathrm{rpm}$. These torque and speed ripples are very small in prediction mode.

Disturbance rejection properties of the drive are investigated next. Previous steady state is the one of Figure 7 (no-load operation at $1200 \mathrm{rpm}$ ) and a load torque equal to the motor rated torque is applied in a step-wise manner at $t=1 \mathrm{~s}$. Responses are shown in Figure 8, for simulation mode and prediction mode. Application of the load torque causes an inevitable dip in speed, of the order of $20 \mathrm{rpm}$ in this period. Motor torque quickly follows the torque reference and enables rapid compensation of the speed dip (in less than $100 \mathrm{~ms}$ ). The motor torque settles at the value equal to the load torque after around $100 \mathrm{~ms}$ and the motor current becomes rated at the end of the transient. The maximum ripples in the torque and speed during this period in simulation mode are 6 $\mathrm{N}-\mathrm{m}$ and $13 \mathrm{rpm}$ and in prediction mode are $0.4 \mathrm{~N}-\mathrm{m}$ and $2 \mathrm{rpm}$. Due to presence of high ripples in torque and speed, the machine produces more noise; more losses, more heating and therefore derating of machine will take place. 

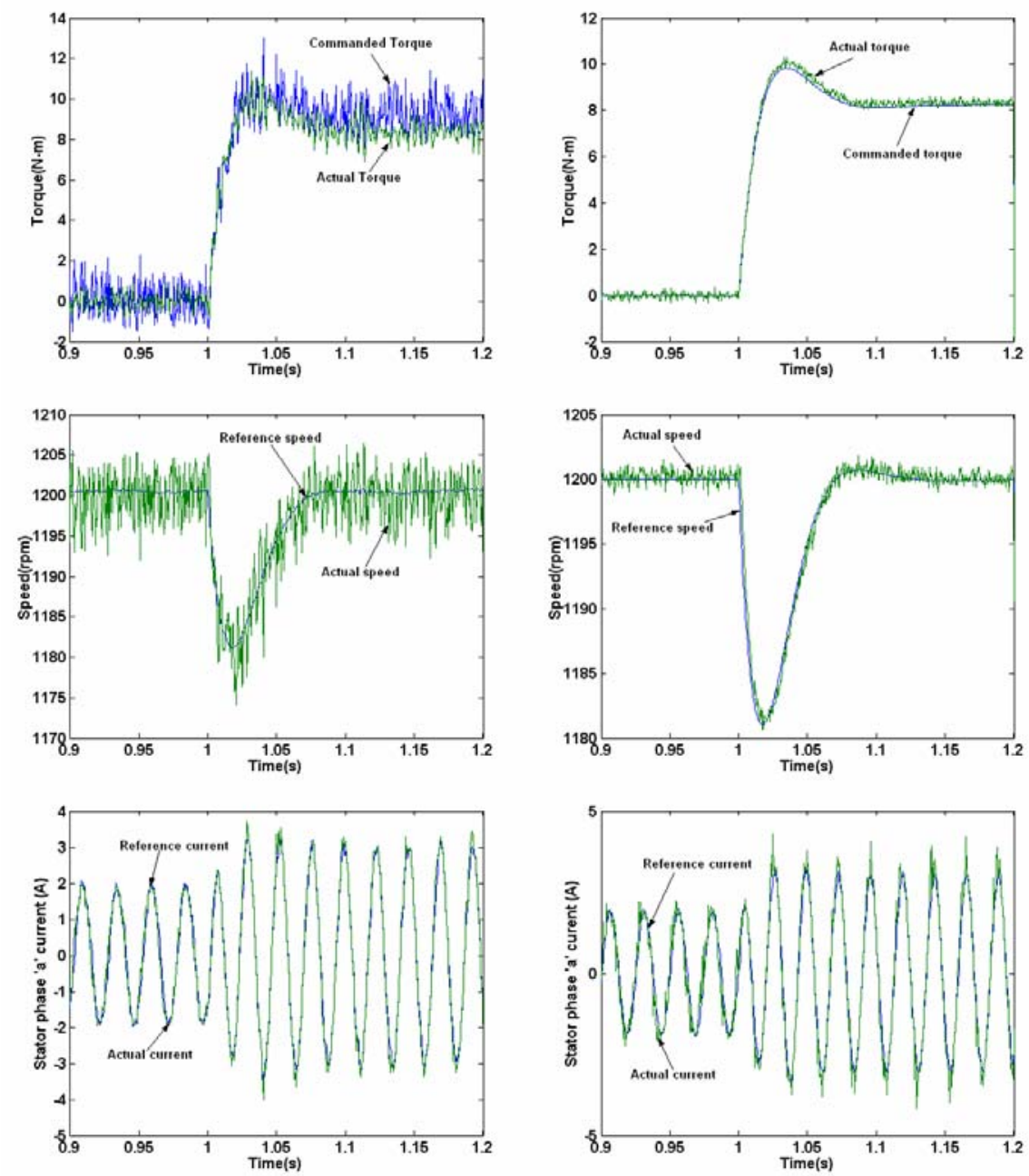

(a)

(b)

Figure 8. The torque, speed and phase 'a' current of a vector controlled five-phase induction machine: disturbance rejection behaviour for (a)simulation mode and(b) prediction mode.

Finally, reversing transient is examined as well. Previous steady state is the one of Figure 8, (rated load torque operation at 1200 $\mathrm{rpm}$ ) and the command for speed reversal is given at $t=1.2 \mathrm{~s}$. Responses, obtained for this transient, are shown in Figure 9, for simulation mode and prediction mode. Once more, actual torque closely follows the reference, leading to the speed reversal, with torque in the limit, in the shortest possible time interval (approximately $350 \mathrm{~ms}$ ). During this period, in simulation mode, the ripple in the torque is $4 \mathrm{~N}-\mathrm{m}$ and a spike in the speed is $200 \mathrm{rpm}$. The torque and speed ripples are very small in prediction mode.

The speed feedback signal is the estimated one obtained from BPN-MRAS-based speed estimator first from simulation mode and then from prediction mode. The attainable performances are examined by simulation in both modes and compared. It is concluded and shown that the performance achieved with prediction mode are better than simulation mode.

\section{(B) Using Modified Euler Integration Method}

This is the improvement of the artificial-neural-network (ANN)-based speed estimator MRAS ANN BPN presented in previous section. In this modified scheme the number of inputs to the adaptive model is increased with a consequent quicker convergence of the speed estimation. This modified ANN MRAS BPN speed estimator uses the current model as an adaptive model discretized with the modified Euler integration method. A neural network has been then designed and trained online by means of a nonlinear back propagation network (BPN) algorithm. Moreover, the neural adaptive model is employed here both in simulation and prediction mode. The quick convergence of the speed estimation \& lower estimation errors both in transient and steady-state operation is obtained in the prediction mode. 

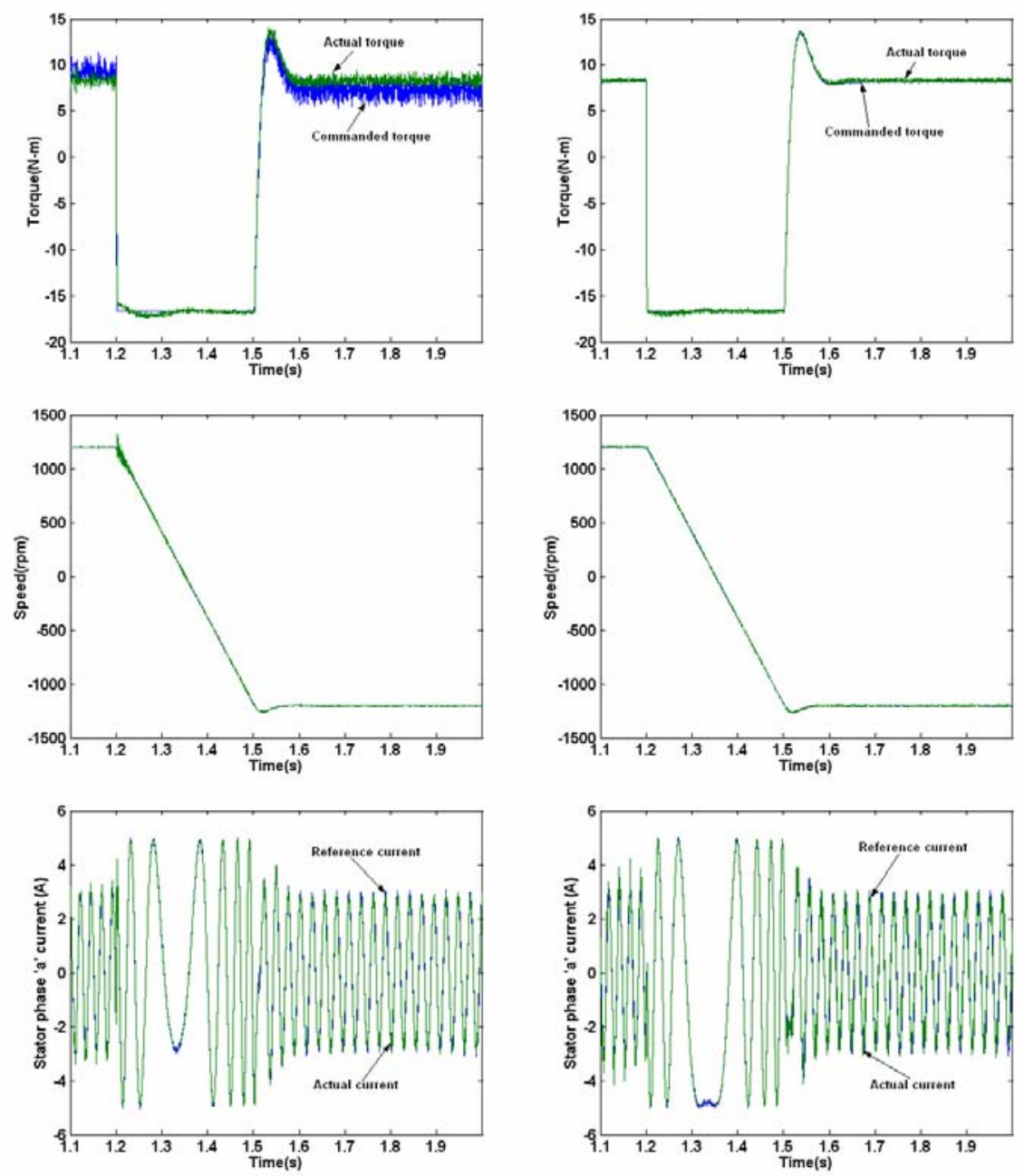

(a)

(b)

Figure 9. The torque, speed and phase 'a' current of a vector controlled five-phase induction machine: reversing transient for (a) simulation mode and (b) prediction mode.

A more efficient integration method is used by the so-called modified Euler integration, which also takes into consideration the values of the variables in two previous time steps.

$$
\begin{aligned}
& \hat{\psi}_{d r}=\frac{1}{T_{r}}\left[\int\left(L_{m} i_{d s}-\hat{\psi}_{d r}-\omega_{r} T_{r} \hat{\psi}_{q r}\right) d t\right] \\
& \hat{\psi}_{q r}=\frac{1}{T_{r}}\left[\int\left(L_{m} i_{q s}-\hat{\psi}_{q r}+\omega_{r} T_{r} \hat{\psi}_{d r}\right) d t\right]
\end{aligned}
$$

From equations (19) \& (20) discrete-time equations (21) can be obtained, as given below:

$$
\begin{aligned}
& \hat{\psi}_{d r}(k+1)=\omega_{1} \hat{\psi}_{d r}(k)-\omega_{2} \hat{\psi}_{q r}(k)+\omega_{3} i_{d s}(k)+\omega_{4} \hat{\psi}_{d r}(k-1)+\omega_{5} \hat{\psi}_{q r}(k-1)-\omega_{6} i_{d s}(k-1) \\
& \hat{\psi}_{q r}(k+1)=\omega_{1} \hat{\psi}_{q r}(k)+\omega_{2} \hat{\psi}_{d r}(k)+\omega_{3} i_{q s}(k)+\omega_{4} \hat{\psi}_{q r}(k-1)-\omega_{5} \hat{\psi}_{d r}(k-1)-\omega_{6} i_{q s}(k-1)
\end{aligned}
$$

where $\omega_{1}, \omega_{2}, \omega_{3}, \omega_{4}, \omega_{5}, \omega_{6}$ are the weights of the neural networks defined as:

$$
\begin{aligned}
& \omega_{1}=1-3 T / 2 T_{r}, \quad \omega_{2}=3 \omega_{r} T / 2, \\
& \omega_{3}=3 L_{m} T / 2 T_{r}, \quad \omega_{4}=T / 2 T_{r}, \\
& \omega_{5}=\omega_{r} T / 2, \quad \omega_{6}=L_{m} T / 2 T_{r} .
\end{aligned}
$$

And the rest of the equations are same as used in the previous ANN MRAS BPN scheme i.e.

$\varepsilon_{d}=\psi_{d r}-\hat{\psi}_{d r} \quad \varepsilon_{q}=\psi_{q r}-\hat{\psi}_{q r}$ 


$$
\begin{gathered}
\Delta w_{2}(k)=\eta\left[-\varepsilon_{d}(k) \hat{\psi}_{q r}(k-1)+\varepsilon_{q}(k) \hat{\psi}_{d r}(k-1)\right] \\
w_{2}(k)=w_{2}(k-1)+\Delta w_{2}(k)+\alpha \Delta w_{2}(k-1)
\end{gathered}
$$

And the speed is obtained as $\omega_{r}=\frac{W_{2}}{T_{s}}$

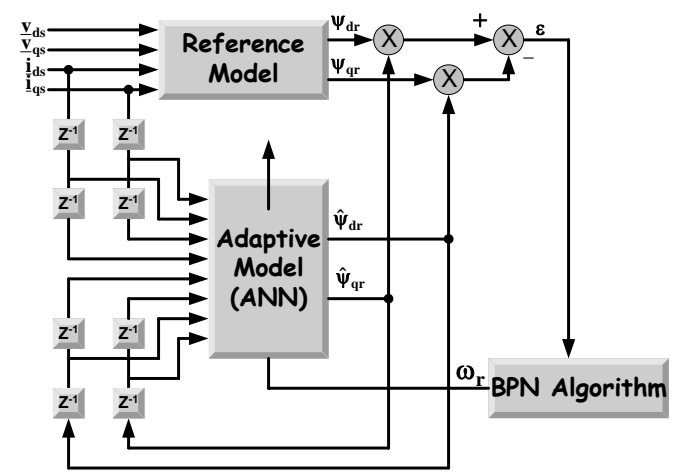

(a)

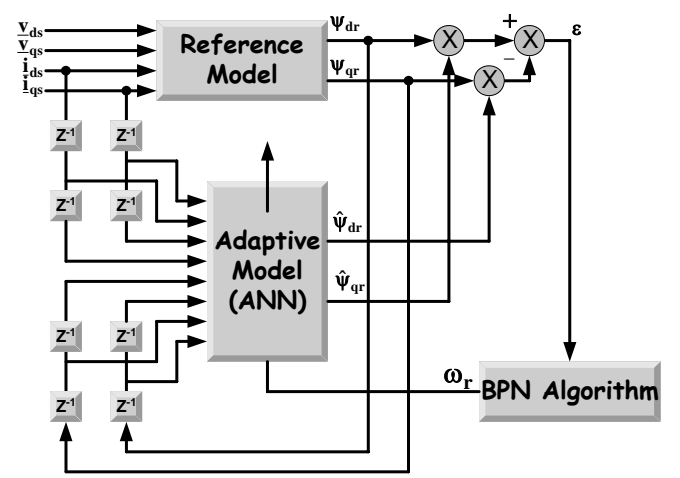

(b)

Figure 10. Schematic block diagram of Recurrent Neural Network speed estimator using modified Euler BPN algorithm in

(a) Simulation mode and (b) Prediction mode

Figure 10 shows the block diagram of the corresponding MRAS speed observer for simulation and prediction mode. The modified Euler has eight inputs and two outputs with a consequent quicker convergence of the speed estimation.

The simple Euler causes an error at starting of transient. This problem can be avoided either by using a simple Euler integration in prediction mode or by using a modified Euler integration either in simulation or in prediction mode. In this last case, however, the use of the prediction mode results in better accuracy in rotor speed estimation and a better performance at zero speed as shown in the Figure 11, and Figure 12.

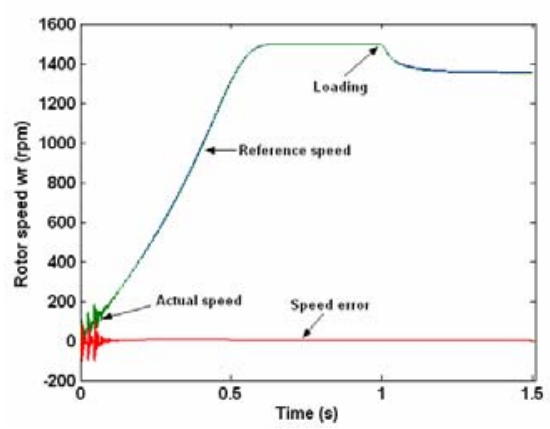

(a)

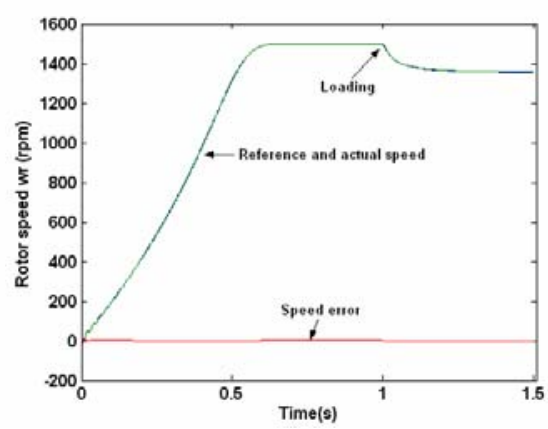

(b)

Figure 11. Speed characteristics of RNN speed estimator using modified Euler BPN algorithm for fixed voltage and fixed frequency supply fed in (a) simulation mode (b) prediction mode.

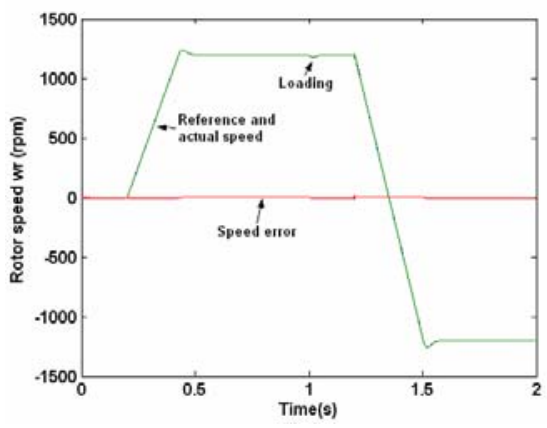

(a)

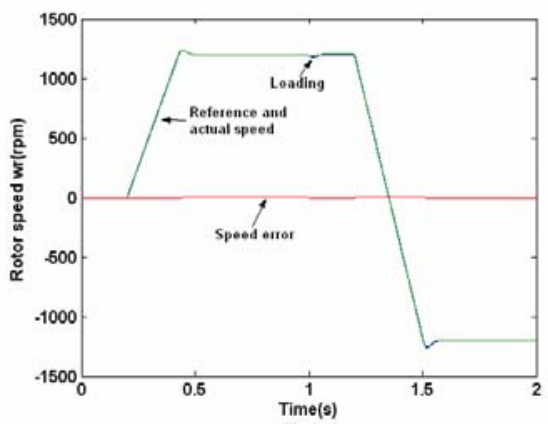

(b)

Figure 12. Speed characteristics of RNN speed estimator using modified Euler BPN algorithm for vector control in

(a) simulation mode (b) prediction mode.

As in the previous BPN scheme (MRAS-ANN-BPN scheme) adaptation algorithm described above is implemented in two ways viz adaptive model in Simulation mode,(ii) adaptive model in prediction mode. 
Figure 11 shows the speed response of the modified ANN MRAS BPN scheme with adaptive model in simulation \& prediction mode and Figure 12 shows the Speed response in presence of a vector controller of the modified ANN-MRASBPN scheme with adaptive model in simulation and prediction mode.

It is clear from the simulation results that much better response is obtained by increasing the number of inputs to the adoptive model. Furthermore the tracking capability further improves. In the simulation mode ripples are found for a period less than 0.1 seconds. After this period the estimator no ripples are there and the estimator closely tracks the actual speed. As shown in Figure 12(b), these starting ripples can be completely eliminated in the prediction mode besides the tracking capability which is much better than with the simulation mode.

\section{Conjugate Gradient Algorithm based speed estimator}

The Conjugate Gradient method is an effective method for symmetric positive definite systems. It is the oldest and best known of the non-stationary methods discussed here. The method proceeds by generating vector sequences of iterates (i.e., successive approximations to the solution), residuals corresponding to the iteration, and search directions used in updating the iterates and residuals. Although the length of these sequences can become large, only a small number of vectors need to be kept in memory. In each iteration of the method, two inner products are performed in order to compute update scalars that are defined to make the sequences satisfy certain orthogonality conditions. On a symmetric positive definite linear system these conditions imply that the distance to the true solution is minimized in some norm.

The bi-conjugate gradient method provides a generalization to non-symmetric matrices. Various nonlinear conjugate gradient methods seek minima of nonlinear equations.

It makes the learning algorithm faster. This method implies that the momentum factor $\alpha$ is changed during learning time according to the following formula:

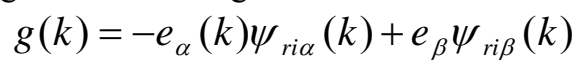

Where, $e(k)=\psi_{r u}(k)-\psi_{r i}(k)$

and $\quad \alpha(k-1)=g^{2}(k) / g^{2}(k-1)$

Figure 13 shows the speed response of the ANN-MRAS-Conjugate Gradient scheme. Small error is present in the transient period but the error is completely omitted in the steady-state region. The conjugate gradient method is faster as compared to the BPN scheme, because the learning rate is modified during learning, thus making the learning algorithm faster.

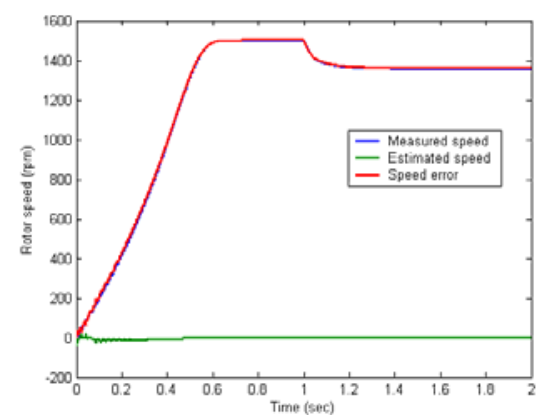

(a)

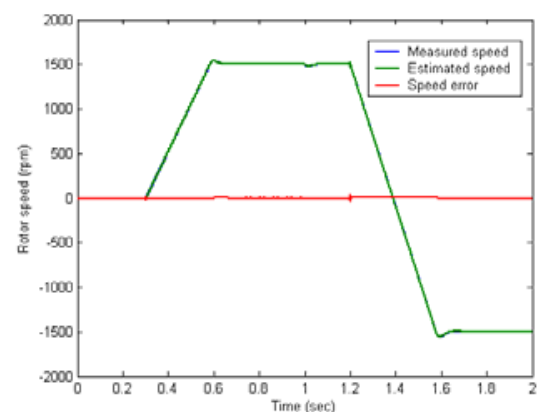

(b)

Figure 13. Speed response of the ANN-MRAS-Conjugate Gradient scheme for (a) fixed voltage and fixed frequency supply (b) with vector controller.

\section{Conclusion}

The paper deals with ANN-MRAS- based sensorless vector control of a five-phase induction machine, utilising an indirect machine is at first reviewed and it is shown that the resulting model is the same as for a three-phase machine rotor flux oriented controller and current control in the stationary reference frame. Hence the same vector control principles and speed estimation technique are applicable for five-phase machine. Operation in the speed mode is further studied, utilising the hysteresis current control. The speed feedback signal is the estimated one obtained from ANN-MRAS-based speed estimator. The attainable performance is examined by simulation. It is shown that the dynamic behaviour, obtainable with the indirect vector control, is the same as it would have been had a three-phase machine been used. Rotor flux and torque control are fully decoupled, enabling the fastest possible accelerations and decelerations with the given torque limit. 


\section{Nomenclature}

$V_{d s}, V_{q s}$ d- and q-axis stator voltages

$i_{d s}, i_{q s}$ d-and q-axis stator currents

$R_{s} \quad$ Stator winding resistance

$L_{s}, L_{s}^{\prime} \quad$ Stator winding self and leakage inductances

$L_{r} \quad$ Rotor winding self inductance

$L_{m} \quad$ Machine mutual inductance

$\psi_{d r}, \psi_{q r} \mathrm{~d}$ - and q-axis rotor fluxes

$\hat{\psi}_{d r}, \hat{\psi}_{q r} \mathrm{~d}$ - and q-axis estimated rotor fluxes

$w_{r}, \hat{w}_{r}$ Actual and estimated rotor speed

$T_{r} \quad$ Rotor time constant

$T_{s} \quad$ Sampling time constant

$\varepsilon_{d}, \varepsilon_{q}$ d- and q-axis rotor fluxes error

$\psi(k) \quad$ kth instant flux

$w_{1}, w_{2}, w_{3}$ ANN weights

$\Delta w \quad$ Weight error

$\eta \quad$ Learning rate

\section{References}

Ben-Brahim L. and R. Kurosawa, 1993. Identification of induction motor speed using neural networks. in Proc. PCC’93,Yokohama, Japan, 1993, pp. 689-694.

Ben-Brahim L., S. Tadakuma, and A. Akdag, 1999. Speed control of induction motor without rotational transducers. IEEE Trans. Ind. Appl., Vol. 35, No. 4, pp. 844-850, Jul./Aug.

Elbulk M. E., L. Tong and I. Husain, 2002. Neural Network based Model Reference Adaptive systems for high performance motor drives and motion controls. IEEE Trans. Ind. Appticat. Vol. 38. May/June.

Elloumi M., L. Ben-Brahim, and M. Al-Hamadi, 1998. Survey of speed sensorless controls for IM drives. in Proc. IEEE IECON'98, Vol. 2, 1998, pp. 1018-1023.

Green S., A.G.Atkinson, B.C.Mecrow and A.King, 2000. Sensorless Operation of a Fault Tolerant PM Drives. IEE Proc. Elect. Power application, 2000, Vol.150, No.2, pp.117-125.

Holtz J., 2006. Sensorless Control of Induction Machines -With or Without Signal Injection?. IEEE Transactions on Industrial Electronics, Vol. 53, No. 1, Feb 2006.

Holtz J., 2002. Sensorless control of induction motors. Proc. IEEE, vol. 90, no. 8, pp. 1358-1394, Aug.

Iqbal A., S.N. Vukosavi and E.Levi, 2003. Vector control of a five-phase induction motor drive. Proc. $38^{\text {th }}$ UPEC, Thessoloniki, Greece, CD-ROM paper No. EMDII, pp.57-60.

Jones M. and Levi E., 2002. A literature survey of state-of-the-art in multiphase ac drives. Proc. $37^{\text {th }}$ Int. Universities Power Eng. Conf. UPEC, Stafford, UK, pp. 505-510.

Kim K. H., S. M. Chung, G. W. Moon, I.C. Baik and M.J. Youn, 1995. Parameter estimation and control for PMSM drive using MRAS. Proc. of 21 ${ }^{\text {st }}$ IEEE IECON, Vol. 1, pp. 387-392, 1995.

Matlab/Simulink reference guide www.mathworks.co.uk

Parsa L., 2005. On Advantages of Multiphase Machines. IECON 2005

Parsa L. and H. Toliyat, 2004. Sensorless Direct Torque Control of Five-Phase Interior Permanent Magnet Motor Drives. IEEE Industry Applications Conference, IEEE-IAS 2004, Annual Meeting ,Oct 3-7.

Rajashekara K., A. Kawamura, and K. Matsuse, 1996. Sensorless Control of AC Motors, Eds. Piscataway. NJ: IEEE Press, 1996.

Shauder C., 1992. Adaptive speed identification for vector control of induction motors without rotational transducers. IEEE Trans. Ind. Appl., Vol. 28, No. 5, pp. 1054-1061, Sep./Oct.

Singh G.K., 2002. Multi-phase induction machine drive research - a survey. Electric Power System Research, Vol. 61, 2002, pp. 139-147. 
Terrien F., S.Siala and P.Noy, 2004. Multiphase Induction Motor Sensorless Control for Electric ship Propulsion. Proc. PEMD 2004.

Toliyat H.A. and Xu H., 2000. DSP-based direct torque control (DTC) for five-phase induction machines. Proc. Int. Power Elec. Conf. IPEC, Tokyo, Japan, pp. 1195-1199.

Vas P., 1998. Sensorless Vector and Direct Torque Control. Oxford University Press.

White D.C., H.H. Woodson, 1959. Electromechanical Energy Conversion. New York, John Wiley \& Sons.

Xu H., Toliyat H.A. and Petersen L.J., 2002. Five-phase induction motors drives with DSP-based control system. IEEE Trans. On Power Electronics, Vol. 17, No. 4, pp. 524-533.

Xu H., H.A. Toliyat and L.J. Petersen, 2002. Resilient current control of five-phase induction motor under asymmetrical fault conditions. Proc. IEEE Applied power Elec. Conf. APEC, Dallas, Texas, pp. 64-71.

\section{Biographical notes}

Dr. M. Rizwan Khan received his B.Sc. Engineering, M.Tech. (Electrical) and Ph.D. degrees in 1998, 2001 and 2008 respectively, from the Aligarh Muslim University, Aligarh, India. He is an Asstt. Professor in the Department of Electrical Engineering, Aligarh Muslim University, Aligarh since 2001. His principal area of research interest is Power Electronics, Artificial Intelligence and Multi-phase motor drives.

Dr. Atif Iqbal received his B.Sc. and M.Sc. Engineering (Electrical) degrees in 1991 and 1996, respectively, from the Aligarh Muslim University, Aligarh, India and $\mathrm{PhD}$ in 2005 from Liverpool John Moores University, Liverpool, UK. He has been employed as Lecturer in the Department of Electrical Engineering, Aligarh Muslim University, Aligarh since 1991 and is currently working as a Reader in the same university. He is on academic assignment and working at Qatar University, Qatar, He is recipient of Maulana Tufail Ahmad Gold Medal for standing first at B.Sc. Engg. Exams in 1991, AMU and research fellowship from EPSRC,UK for pursuing Ph.D. studies. His principal area of research interest is Power Electronics and Multi-phase machine drives.

Sk. Moin Ahmed was born in Hooghly, West Bengal, India, in 1983. He received the B.Tech and M.Tech. degrees from Aligarh Muslim University (AMU), Aligarh, India, in 2006 and 2008, respectively, where he is currently pursuing the Ph.D. degree. He is also pursuing a research assignment at Texas A\&M University at Qatar. His principal areas of research are modeling, simulation, and control of multi-phase power electronic converters and fault diagnosis using Artificial intelligence. Mr. Ahmed was a Gold Medalist in earning the M.Tech degree. He is a recipient of Toronto Fellowship, funded by AMU.

Saifullah Payami received his B.Tech (Electrical) and M.Tech (Power System \& Drives) in 2007 and 2010, respectively, from Aligarh Muslim University, Aligarh, India. Currently he is working as Junior Research Fellow in a CSIR funded project at Department of Electrical Engineering, AMU. His area of research interest is Power Electronics, Multi-phase Machine Drives and Renewable Energy Systems

Received August 2010

Accepted October 2010

Final acceptance in revised form November 2010 